\title{
BMJ Open Strategies to improve the implementation of policies, practices or programmes in sporting organisations targeting poor diet, physical inactivity, obesity, risky alcohol use or tobacco use: a systematic review
}

\author{
Tameka McFadyen, ${ }^{1,2}$ Li Kheng Chai, ${ }^{3}$ Rebecca Wyse, ${ }^{1}$ Melanie Kingsland, \\ Sze Lin Yoong, ${ }^{1}$ Tara Clinton-McHarg, ${ }^{1}$ Adrian Bauman, ${ }^{4}$ John Wiggers, ${ }^{1,2}$ \\ Chris Rissel, ${ }^{4}$ Christopher Michael Williams, ${ }^{1}$ Luke Wolfenden ${ }^{1,2}$
}

To cite: McFadyen T, Chai LK, Wyse R, et al. Strategies to improve the implementation of policies, practices or programmes in sporting organisations targeting poor diet, physical inactivity, obesity, risky alcohol use or tobacco use: a systematic review. BMJ Open 2018;8:e019151. doi:10.1136/ bmjopen-2017-019151

- Prepublication history and additional material for this paper are available online. To view these files, please visit the journal online (http://dx.doi. org/10.1136/bmjopen-2017019151).

Received 22 August 2017 Revised 19 April 2018 Accepted 22 June 2018

D) Check for updates

(C) Author(s) (or their employer(s)) 2018. Re-use permitted under CC BY-NC. No commercial re-use. See rights and permissions. Published by BMJ.

For numbered affiliations see end of article.

Correspondence to Tameka McFadyen;

Tameka-Rae.Small@hnehealth. nsw.gov.au

\section{ABSTRACT}

Objectives The primary aim for this review is to determine the effectiveness of strategies to improve the implementation of policies, practices or programmes in sporting organisations. The secondary aims are to describe the cost or cost-effectiveness and adverse effects of such strategies and to examine the effects of those implementation strategies on individual's diet, physical activity, obesity, alcohol use or tobacco use.

Methods We conducted searches of academic databases (eg, MEDLINE, EMBASE and CENTRAL), trial registers and hand searches of selected journals. Studies were included if they were conducted at a sporting venue; described a strategy to improve implementation of policies, practices or programmes focusing on one or more health risks (diet, physical inactivity, obesity, alcohol or tobacco use), and included a parallel control group. Two authors independently screened citations and extracted data. The results of included studies were synthesised narratively.

Results Of the 5926 citations screened three studies met the inclusion criteria. Two studies were randomised controlled trials. Two studies sought to improve the implementation of nutrition-related policy and practices and one study sought to improve implementation of alcohol-related policy and practices. Each study reported improvement in at least one measure of policy or practice implementation. Two studies reported individual-level outcomes and found a reduction in excessive alcohol consumption and an increase in purchase of fruits and vegetables at the sports club ground. Two studies assessed club revenue as a potential adverse effect, neither reported significant between-group differences on these measures.

Conclusion There is a sparse evidence base regarding the effectiveness of strategies to improve the implementation of policies, practices or programmes targeting chronic disease risk factors in sporting clubs. While all studies reported some improvements in implementation, for some multistrategic implementation
Strengths and limitations of this study

- This is the first synthesis of implementation studies targeting multiple health risk behaviours in the sports setting.

- All included studies were in high-income countries and had self-reported outcomes.

- The review identified a limited number of studies within this area which met the inclusion criteria.

- As there is considerable heterogeneity in terms used to describe implementation, the search terms may not have identified all potentially eligible trials.

strategies it is difficult to determine the extent to which such effects are generalisable.

PROSPERO registration number CRD42016039490.

\section{INTRODUCTION}

Physical inactivity, poor diet, obesity, tobacco use and risky alcohol consumption are the five most common modifiable risks contributing to the prevalence of chronic disease. ${ }^{1}$ Each risk factor accounts for a significant proportion $(2.78 \%-9.24 \%)$ of the total global disease burden. ${ }^{2}$ In 2010, all risk factors estimated to result in more than 580 million years lived with disability and 24 million deaths. ${ }^{2}$ Consequently, reducing the impact of these modifiable health risks in the population is a public health priority. ${ }^{3}$

The implementation of health promotion interventions in community settings has been recommended by the WHO to reduce these modifiable health risks. ${ }^{3}$ Such an approach is encouraged as settings provide a centralised point to access large numbers of individuals 
for intervention, and the infrastructure of community organisations, such as schools, hospitals, sporting clubs and workplaces, can be used to support intervention delivery. One attractive setting to support risk factor reduction is non-elite community sporting organisations or clubs where organised sport is undertaken. ${ }^{4-7}$ Large numbers of people globally are associated with community sport. For example, between 2015 and 2016 over 15 million people aged 16 years or over $(36.1 \%)$ in England ${ }^{8}$ and over 17 million people aged 15 years and over $(87 \%)$ in Australia ${ }^{9}$ engaged in organised sport and physical activity.

A number of sport and exercise-based interventions have been found to effectively improve weight status, ${ }^{10-12}$ $\operatorname{diet}^{13}$ and physical activity ${ }^{12-14}$ and reduce risky levels of alcohol consumption ${ }^{15}$ and tobacco use ${ }^{16}$ of sports club members and affiliates. As such, best practice guidelines recommend sporting clubs implement a range of policies and practices to create environments more supportive of healthy behaviour. ${ }^{17}{ }^{18}$ Despite such evidence, implementation of recommended health-promoting policies and practices remains limited in this setting. For example, in a cross-sectional study of 88 sports stadia across 10 European countries, only $18 \%$ had a healthy eating policy, $22 \%$ had an initiative to support responsible alcohol use, $50 \%$ had a physical activity promotion policy and $55 \%$ had some form of tobacco control policy. ${ }^{19}$ Poor implementation of health-promoting practices has also been reported in other studies ${ }^{20}$ and settings. ${ }^{21}$

Implementation of effective sporting club public health interventions is required if their potential benefits to community health are to be realised. However, there remains little evidence to guide implementation efforts of governments, sporting associations and club officials. A systematic review, conducted by the Agency for Healthcare and Research Quality (AHRQ), investigated the effectiveness of strategies in any community setting, inclusive of sports and recreational clubs, to implement policies or practices to reduce behavioural risks for cancer, including healthy eating, physical activity, tobacco use and sun protection. ${ }^{22}$ The review, which included studies published between 1980 and 2008, failed to identify any implementation study targeting these risks in the sports club settings. We are not aware of any reviews of implementation strategies in this setting since the AHRQ review. As such, an updated synthesis of the evidence is warranted.

\section{OBJECTIVES}

The primary aim of this review is to determine the effectiveness of strategies to improve the implementation of policies, practices or programmes in sporting organisations targeting poor diet, physical inactivity, obesity, risky alcohol use or tobacco use.

The secondary aims of the review are to:

- Describe the cost or cost-effectiveness of such implementation strategies.
- Examine the effects of such strategies on diet, physical activity, obesity, alcohol use or tobacco use.

- Describe any adverse effects of such strategies on sporting organisations, staff, players or spectators.

\section{METHODS}

The review was undertaken according to the methods prescribed in the Cochrane Handbook for Systematic Reviews of Interventions ${ }^{23}$ and is reported according to the Preferred Reporting Items for Systematic Reviews and Meta-Analyses. ${ }^{24}$ The review objectives and methods were registered with PROSPERO.

\section{Eligibility}

Types of studies

Any study with a parallel control group (a group participating in study at the same time as the intervention group, but receiving no or a modified form of intervention) was eligible including:

- Randomised controlled trials (RCT) and cluster RCTs.

- Quasi-RCTs/pseudo-RCTs and cluster quasi-RCTs/ pseudo-RCTs.

- Controlled before-and-after studies (CBA) and cluster CBAs.

- Time series designs and parallel controlled trials. Studies were included only if they:

1. Compared a strategy to improve implementation of policy, practice or programme focusing on one (or multiple) of the following risks: diet, physical activity, obesity, alcohol or tobacco use at a sporting venue with no intervention or 'usual practice' comparison; or

2. Compared two or more strategies to improve implementation of diet, physical activity, obesity, alcohol or tobacco use policy, practice or programme at a sporting venue.

Studies could be published in any language or conducted in any geographic region. Studies were excluded that did not report baseline measures of the primary outcome.

\section{Types of participants}

Studies conducted in any organisation that is a venue to undertake organised sport were included. This could include non-elite community sports clubs, recreational centres, as well as professional/elite sporting clubs and stadia. Participants may include sporting organisation managers or executive, staff, player or others at any level of the organisation, or other organisations which may influence the implementation of health-promoting programmes, practices or policies in this setting.

\section{Types of interventions}

Any intervention with the intent of improving implementation of a policy, practice or programme targeting diet, physical inactivity, obesity, risky alcohol use or tobacco use was included. This could include, for example, quality improvement initiatives, education and training, performance feedback, prompts and reminders, implementation resources, financial incentives, penalties, communication and social marketing strategies, professional networking, 
the use of opinion leaders or implementation consensus processes,${ }^{25}$ or a combination of strategies.

Types of outcome measures

\section{Primary outcomes}

The primary outcomes could include any measure of the implementation of policy, practice, or programme targeting poor diet, physical inactivity, obesity, risky alcohol use or tobacco use. For example, percentage of sporting clubs implementing a recommended policy or practice or the mean number of health-promoting practices implemented by a sporting organisation. Data on these outcomes could be obtained from self-report measures (eg, completed by club officials), direct observation by researchers, audits of organisational records, audits of data collected by external organisations (eg, parent company, government) or other methods.

\section{Secondary outcomes}

1. Estimates of absolute costs or any assessment of the cost-effectiveness of strategies to improve the implementation of policies, practices or programmes in sports clubs.

2. Any measure of diet, physical activity (including sedentary behaviours), weight status, alcohol or tobacco use. Such measures could be derived from any data source including direct observation, questionnaire, or anthropometric or biochemical assessments. Studies focusing on malnutrition/malnourishment were excluded.

3. Any reported adverse consequences of a strategy to improve the implementation of policies, practices or programmes in sports clubs.

\section{Search methods for identification of studies}

Searches for peer-reviewed literature were performed in electronic databases, by hand searching of relevant journals and the reference lists of included trials and searches of the web. Searches for grey literature were also conducted in the same way, with a focus on web-based search engines and government websites.

\section{Electronic searches}

The following electronic databases were searched:

- MEDLINE and MEDLINE In-Process and Other Non-Indexed Citations (1946 to May 2016).

- EMBASE (1974 to May 2016).

- PsycINFO (1806 to May 2016).

- CiNAHL (1981 to May 2016).

- SPORTDiscus (1973 to May 2016).

- Dissertation Abstracts (1997 to May 2016).

- Sociological Abstracts (1952 to May 2016).

- Cochrane Central Register of Controlled Trials (CENTRAL) (up to 2016).

The MEDLINE search strategy described in online supplementary appendix 1 was used and adapted for use in the other above-mentioned databases. Search filters used in other reviews were employed for organised sport venues including professional elite sporting clubs and stadia, ${ }^{26}$ physical activity, ${ }^{26}$ healthy eating, ${ }^{27-29}$ obesity, ${ }^{30}$ tobacco use prevention ${ }^{31}$ and alcohol misuse. ${ }^{32}$ Additionally, for intervention/implementation strategies, search filters employed in a previous Cochrane Review ${ }^{21}$ and originally developed based on common terms in implementation and dissemination research ${ }^{22} 33$ were used.

\section{Searching other resources}

The reference lists of all included trials were searched for other potentially eligible studies. Hand searches of all publications for the past 5 years in the journals: Implementation Science and the Journal of Translational Behavioural Medicine were conducted. Searches of the WHO International Clinical Trials Registry Platform (www.who. int/ictrp/), WHO European database on Nutrition, Obesity and Physical Activity (http://data.euro.who.int/ nopa/) and ClinicalTrials.gov (www.clinicaltrials.gov) were conducted to identify any studies in progress or completed that may be eligible. Contact was also made with the authors of included trials and experts in the field of implementation science to identify any relevant ongoing or unpublished trials or grey literature publications. All contacted authors and implementation science experts responded, with no additional eligible studies.

\section{Data collection and analysis}

\section{Selection of studies}

Two review authors (LKC and RW) independently screened abstracts and titles for potentially eligible studies. Review authors were not blind to author or journal information. Screening was performed using a standardised screening tool developed based on the Cochrane Handbook for Systematic Reviews of Interventions. ${ }^{23}$ The tool, which has previously been used by the author team in another systematic review, ${ }^{21}$ was adapted for relevance to the setting of this review and piloted before use. The full texts of potentially eligible trials were obtained for further examination. Discrepancies between review authors regarding study eligibility were resolved by consensus, or when required, by a third review author.

\section{Data extraction and management}

Two review authors (LKC and RW) independently extracted information from the included trials. Review authors were not blind to author or journal information. Data were extracted using a form that was developed based on recommendations of the Cochrane Public Health Group Guide for Developing a Cochrane Protocol (Cochrane Public Health Group 2011). The form, which was previously used by the author team in other systematic reviews, ${ }^{21}{ }^{34}$ was adapted for use in this review and was piloted before use. Any discrepancies between review authors regarding data extraction were resolved by consensus and, where required, via a third review author.

Specifically the following information was extracted:

1. Study eligibility, study design, date of publication, sports club/organisation, country, participant/service demographic/socioeconomic characteristics and number of experimental conditions, and information to allow assessment of study risk of bias. 
2. Characteristics of the implementation strategy, including the duration, number of contacts and approaches to implementation, the theoretical underpinning of the strategy (if noted in the study), information to allow classification against the Cochrane Effective Practice and Organisation of Care (EPOC) taxonomy (see online supplementary appendix 2 for definitions) and to enable an assessment of the overall quality of evidence using the Grades of Recommendation, Assessment, Development and Evaluation (GRADE) approach, ${ }^{35}$ and data describing consistency of the execution of the intervention with a planned delivery protocol.

3. Trial primary and secondary outcomes, including the data collection method, validity of measures used, effect size and measures of outcome variability.

4. Source(s) of research funding and potential conflicts of interest.

\section{Assessment of risk of bias in included studies \\ Overall risk of bias}

Three review authors (TM, LKC and TCM) assessed risk of bias independently, using the 'Risk of bias' tool described in the Cochrane Handbook for Systematic Reviews of Interventions. ${ }^{23}$ A risk of bias classification ('high', 'low' or 'unclear') was assigned for each of the following study characteristics: sequence generation, allocation concealment, blinding of participants and personnel, blinding of outcome assessment, incomplete outcome data, selective outcome reporting and 'other' potential sources of bias. Additionally, a criterion for recruitment bias, baseline imbalances, loss of clusters, incorrect analysis, contamination and compatibility with individually randomised trials was included for the assessment of the risk of bias in cluster trial designs. ${ }^{23}$ An overall risk of bias was assigned to each study giving consideration to all such study characteristics. The risk of bias of the included studies is documented in 'Risk of bias' table (figure 1).

\section{Measures of treatment effect}

Differences in measures of primary and secondary outcomes reported in included studies precluded the use of summary statistics to describe treatment effects. As such, a comprehensive description of the methods and outcomes of included trials is described narratively according to broad implementation strategy characteristics.

\section{Unit of analysis issues \\ Clustered studies}

All clustered trials were examined for unit of analysis errors. Where they occur, unit of analysis errors were documented in the 'Risk of bias' table (figure 1).

\section{Assessment of heterogeneity}

Quantitative assessment of heterogeneity was not performed due to variance in reported outcomes, study interventions, measures and population groups. Thus, box plots, forest plots and/or the $\mathrm{I}^{2}$ statistics were also not

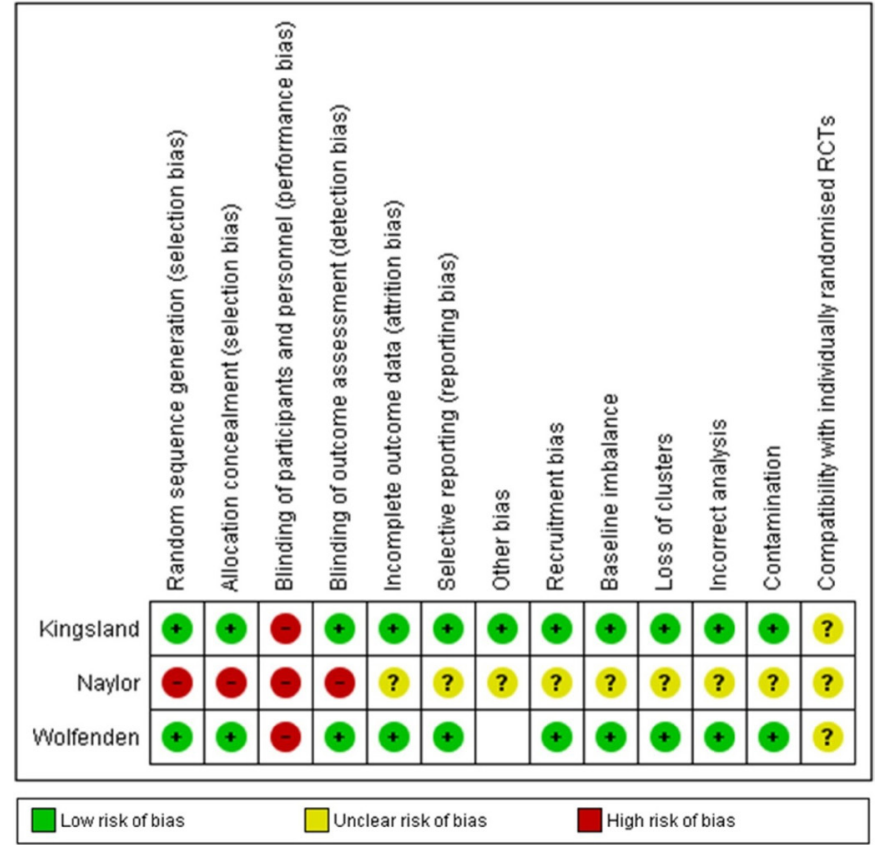

Figure 1 Risk of bias graph, reviewing authors' judgements about each risk of bias item across all included studies, classifying as low risk, unclear risk or high risk.

performed to explore heterogeneity. ${ }^{23}$ Instead heterogeneity was described narratively.

\section{Assessment of reporting biases}

Published reports with trial protocols and trial registers were compared where such reports are available. Occurrences of potential reporting bias were documented in the 'Risk of bias' table if identified.

\section{Data synthesis}

Study characteristics were grouped as types of studies, participants and implementation strategies. Implementation strategies were classified using the EPOC taxonomy. ${ }^{25}$ As trial heterogeneity precluded meta-analysis the trial findings were described and synthesised narratively. The primary outcome (effectiveness of strategies to improve implementation) and secondary outcomes (cost or cost-effectiveness of intervention strategies, effects on poor diet, physical inactivity, obesity, risky alcohol use or tobacco use and reported adverse consequences) for the review reported all available and applicable statistical and descriptive data of the included studies. The GRADE system $^{35}$ was used by two reviewers (TM, LW) to assess the quality of the body of evidence through consideration of study limitations, consistency of effect, imprecision, indirectness and publication bias.

\section{Patient and public involvement}

Neither patients nor members of the general public were involved in this study as it was a systematic review of existing studies. 


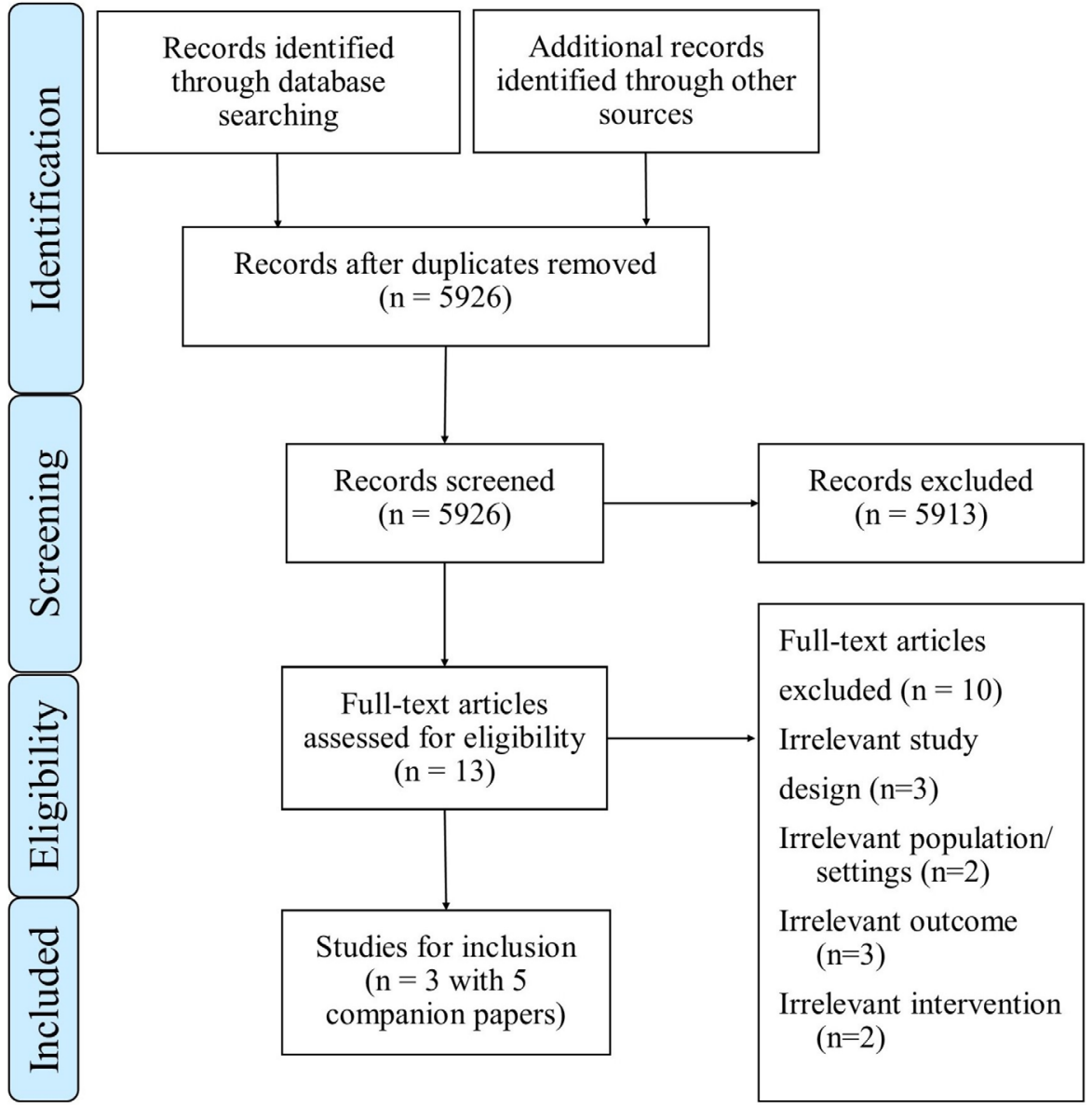

Figure 2 Preferred Reporting Items for Systematic Reviews and Meta-Analyses (PRISMA) flow diagram, depicting the process undertaken for the review with the inclusion of the number of studies that were screened and assessed for eligibility.

\section{RESULTS}

\section{Results of the search}

The searches generated 5867 citations (following duplicate removal) with the addition of 59 citations identified from other sources $(n=5926)$. Screening of titles and abstracts identified 13 manuscripts for full-text review (excluded 5913). The more common reasons for exclusion included: study design, setting and study participant. Of these, three trials (Kingsland $e t a l^{6}$, Wolfenden $e t a l^{37}$ and Naylor $e t a l^{38}$ ) met the inclusion criteria (figure 2). Given the limited number of included trials, the study inclusion criteria were relaxed and citations re-examined by two authors (TM and RW) to identify pre-post trials without a parallel control group, however no additional trials were found to be eligible.

\section{Excluded studies}

Of the 10 excluded papers, three were deemed ineligible based on study design characteristics, two due to population/setting characteristics, three based on study outcomes and two based on intervention characteristics (figure 2).

\section{Characteristics of included studies}

A description of the included studies is presented in table 1. Five companion papers were found for the included studies: four papers for Kingsland $e t a l^{36}$ and one paper for Naylor et al. ${ }^{38}$ All companion papers were reviewed for information relevant to the review, and data extracted from such texts when appropriate. The companion papers included study protocols, secondary outcomes, prevalence studies and an economic report.

Types of studies

Of the three included studies, two were conducted in Australia (Kingsland $e t a l^{36}$ and Wolfenden $e t a l^{37}$ ). These two trials shared similar infrastructure, with the trial conducted by Wolfenden et al nested within the trial by Kingsland $e$ al. The remaining study was conducted in Canada (Naylor $e t a l^{88}$ ). Studies took place between 2009 and 2012. The sample sizes ranged from 85 sports clubs ${ }^{36}$ to 106 recreation and sports facilities. ${ }^{38}$ The studies by Kingsland $e t$ al and Wolfenden et al used an RCT design and the study by Naylor et al used a quasiexperimental, controlled, pre-post comparisons design. Kingsland et al aimed to assess the effectiveness of an intervention to increase the implementation of alcohol management practices, while Wolfenden et al sort to assess the effect of an intervention on (1) the availability of fruit and vegetable and non-sugar-sweetened drink products; (2) the promotion of fruit and vegetable and non-sugar-sweetened drink 


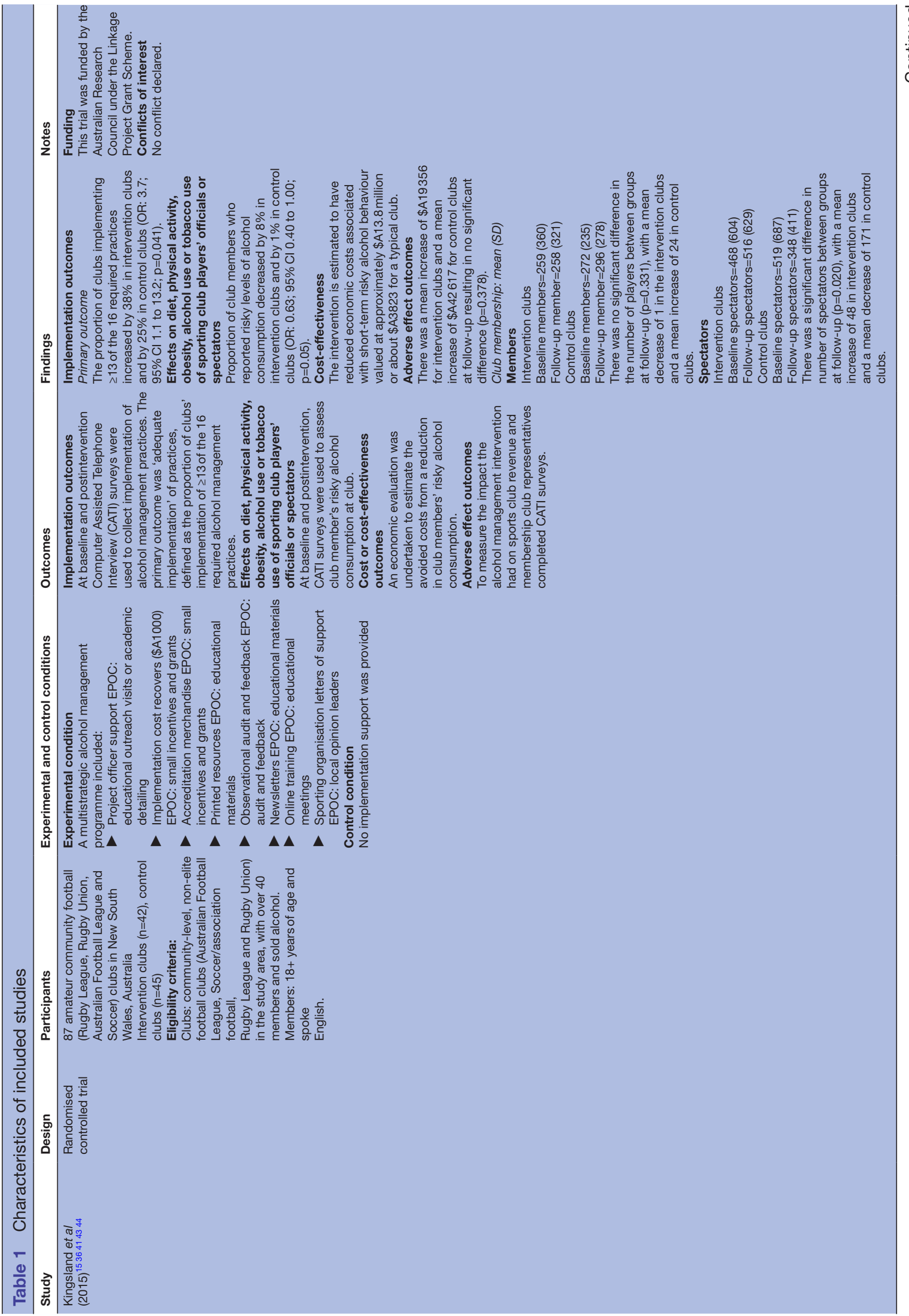




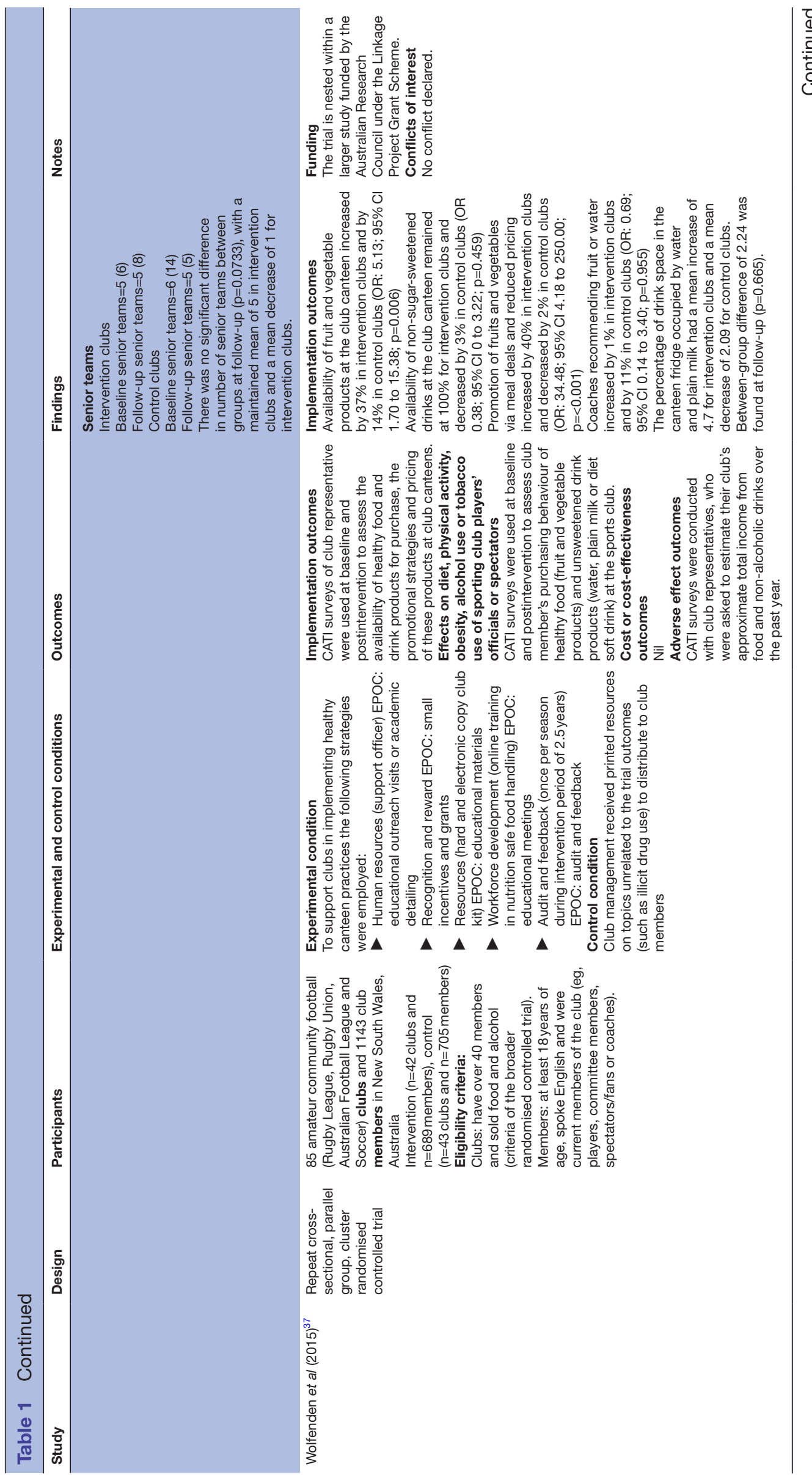




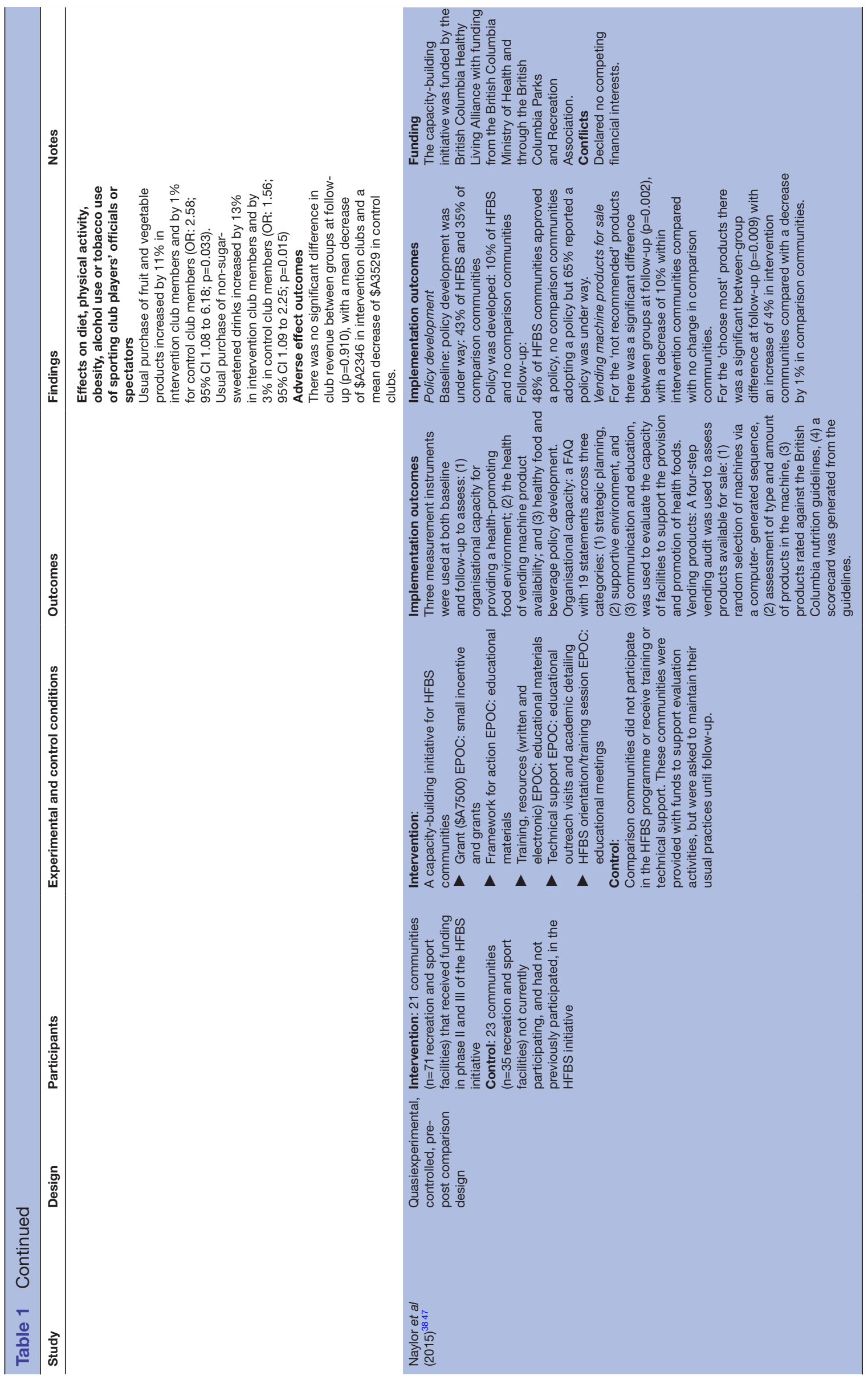

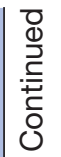




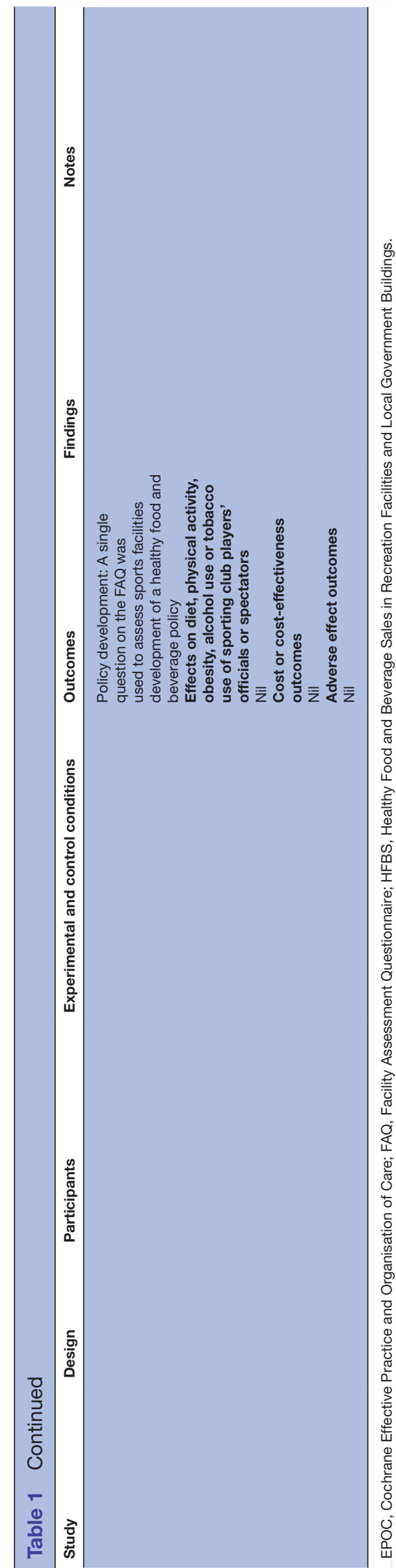

products; and (3) sporting club member purchasing fruit and vegetable and non-sugar-sweetened drink products from community sporting club canteens. Naylor et al sort to determine the effectiveness of an intervention on (1) sports facility organisational capacity for providing a health-promoting food environment; (2) vending products offered for sale; and (3) food policy development.

\section{Participants}

Community-level football clubs from the state of New South Wales, Australia, were recruited for Kingsland et $a l^{36}$ and Wolfenden $e t a l^{37}$ The majority of these clubs were from the Rugby League and Rugby Union football codes, were located in major city areas and had over 160 registered players. The Naylor et al's study recruited recreation and sport facilities within communities from British Columbia, Canada. ${ }^{38}$ The type of recreation and sport facilities varied within communities including: outdoor sporting facilities, pools, gyms, ice rinks, curling rinks and multiplexes.

\section{Implementation strategies}

Strategies to support implementation of the targeted policy, programmes or practices varied from 8 months, ${ }^{38}$ 2 years ${ }^{36}$ and $2 \frac{1}{2}$ years. $^{37}$ All three study interventions employed multiple implementation strategies. The implementation strategies used by the included trials are described below and classified according to EPOC taxonomy. ${ }^{25}$ The definitions for the EPOC categories which classify the implementation strategies outlined in this review can be found in online supplementary appendix 2. All three studies included the use of personal support and educational material (resource kits). Two of the three studies used audit and feedback methods, online training and education. ${ }^{36}{ }^{37}$ All studies used monetary incentives. ${ }^{36-38}$

Kingsland $e t a l^{36}$ aimed to increase the implementation of responsible alcohol management practices in community sports clubs. The implementation intervention was based on theoretical frameworks for organisational change. ${ }^{39}{ }^{40}$ Clubs were required to implement the following alcohol management practices as per the published protocol ${ }^{41}$ : bar servers do not consume alcohol while on duty; substantial food is provided when alcohol is served; non-alcoholic drink options are available; low-alcoholic drink options are available; low-alcoholic drink options are cheaper than full strength; a club committee member is always present when alcohol is served; all bar staff are trained in responsible service of alcohol; an alcohol incidents register is maintained; written alcohol management and safe transport policies are maintained; and no drinking games or promotions are permitted/ conducted. The implementation of these practices was supported via a multistrategy implementation intervention. Strategies included: educational outreach visits or academic detailing (project officer support), small incentives or grants (implementation cost recovery; accreditation merchandise, recognition through an accreditation 
framework), education material (printed resources and newsletters), educational meetings (online training), audit and feedback (observational audit and feedback) and local opinion leader support (sporting organisation letters of support). No implementation strategies were provided to control clubs.

The study by Wolfenden $e t a l^{37}$ aimed to increase the availability and promotion of non-sugar-sweetened drinks, fruit and vegetable products within community sports club canteens. The intervention period occurred over two and a half Australian winter sporting seasons. The social-ecological model of health ${ }^{42}$ was used to inform the development of the intervention. This study sought to improve the implementation of the following practices: providing a total of six fruit and vegetable and non-sugar-sweetened drink products; ensuring that at least $75 \%$ of non-alcohol drinks were non-sugar sweetened; encouraging the purchase of health food and beverages through meal deals; using pricing strategies to encourage sales of these products; displaying these products in prominent positions at the canteen; engaging club coaches and having them recommend consumption of healthy food and beverages to the players during half-time and after games; and development of a written food and nutrition policy and improve club member awareness and attitudes regarding health foods and beverages via information distribution. To facilitate implementation of these practices, strategies included: educational outreach visits or academic detailing (support officers), small incentives or grants (accreditation merchandise; recognition through an accreditation framework), educational materials (hard copy and electronic resource kit), educational meetings (online nutrition and safe food handling training) and audit and feedback (audit and feedback on practice implementation). Control clubs received educational materials (printed resources on topics unrelated to the trial outcome, such as illicit drug use).

Naylor $e t a l^{38}$ aimed to support communities in improving the food environment of recreation and sports facilities via the implementation of the Healthy Food and Beverage Sales in Recreation Facilities and Local Government Buildings (HFBS) initiative. The HFBS initiative adopted a capacity-building approach and used a framework for action. ${ }^{38}$ Sporting facilities were assessed on their implementation of healthy food and beverage practices and policy via a Facility Assessment Questionnaire (FAQ) and a four-step vending audit. The implementation of the HFBS initiative was supported by strategies such as: small incentives and grants (grant of \$C7500), educational materials (planning tools; written and electronic resources; a framework for action), educational meetings (training sessions) and educational outreach visits or academic detailing (technical support via face-to-face, monthly and ad hoc telephone meetings or website). A representative from intervention communities received specific training on the HFBS model, the use of the study's FAQ and four-step vending audit, goal setting and policy development information and stakeholder presentations.
Comparison communities did not receive support or training, or participate in the HFBS initiative; they were encouraged to maintain usual practice. However, they were provided with funds to support evaluation activities.

\section{Quality of the evidence}

Overall, the quality of the body of evidence in the review was rated as very low across all GRADE domains ${ }^{35}$ and in accordance with the Cochrane Handbook for Systematic Reviews of Interventions, ${ }^{23}$ suggesting that the effects of interventions reported in the review may differ from the true effects.

\section{Risk of bias}

The level of risk of bias for each study is presented in figure 1. Justification for the risk of bias assessment is presented in online supplementary appendix 3. Risk of bias was considered to be high for performance bias across all studies. For Kingsland $e t a l$ and Wolfenden $e t$ $a l$, all other categories were considered to have low risk of bias, while Naylor $e t a l$ 's study was considered to have high risk of bias for selection bias and detection bias, with risk of bias considered to be unclear across all other categories.

\section{Primary outcomes}

Effectiveness of strategies to improve implementation

The review findings regarding the effectiveness of strategies to improve implementation were mixed. Kingsland et $a l^{36}$ found a significant increase of $38 \%$, relative to control, in the proportion of intervention clubs implementing 13 or more of 16 alcohol management practices $(\mathrm{p}=0.04)$, at follow-up.

Wolfenden $e t a l^{37}$ found significant improvements in the proportion of intervention clubs promoting healthier food and beverage options through meal deals and reducing prices of fruit and vegetable products, compared with control clubs $(\mathrm{OR}=34.48$; $95 \%$ CI 4.18 to 250.00) after intervention. A significant increase in the availability of fruit and vegetable products at sports club canteens was also found for intervention clubs $(37 \%)$ relative to control clubs (14\%) (OR=5.13; 95\% CI 1.70 to $15.38 ; \mathrm{p}=0.006)$. However, there was no significant difference between groups after intervention for the availability of non-sugar-sweetened drinks ( $\mathrm{OR}=0.38$; $95 \%$ CI 0 to 3.22; $\mathrm{p}=0.459)$, coaches recommending fruit or water $(\mathrm{OR}=0.69 ; 95 \% \mathrm{CI} 0.14$ to $3.40 ; \mathrm{p}=0.955)$ and the percentage of drink space in fridge occupied by water or plain milk (mean difference 2.24; $\mathrm{p}=0.665$ ).

Finally, Naylor et $a l^{88}$ found, at follow-up, intervention communities had a significantly higher increase in FAQ score overall (mean (SD) $=12.70(9.02)$ ) compared with comparison communities (mean $(\mathrm{SD})=3.06(4.16)$ ) $(p=<0.001)$. At baseline $10 \%$ of intervention communities reported having a policy in place, compared with no comparison communities. At follow-up, there was an increase of $38 \%$ of intervention communities having a policy in place, there was no change in the number of 
comparison communities. Significant between-group differences $(p=0.002)$ were found at follow-up, with intervention communities decreasing the proportion of 'not recommended' products in vending machines by $10 \%$, with no change in comparison communities. Furthermore, significant between-group differences $(p=0.009)$ were found for the 'choose most' products in vending machines with intervention communities increasing the proportion of the category by $4 \%$, whereas a decrease of $1 \%$ was found in comparison communities.

\section{Secondary outcomes}

Table 1 summarises the effects of each study.

\section{Cost or cost-effectiveness of intervention strategies}

An economic evaluation was undertaken for Kingsland $e t$ $a l$ 's study. ${ }^{43}$ The estimated avoided costs from a reduction in club members' risky alcohol consumption were used to calculate the potential benefits of this study intervention. Estimated costs included: road accidents, falls and assaults for club members between the ages of 18 and 30 years, and attributing part of these costs to risky drinking using attributable fractions. Net benefits of the intervention were calculated by comparing the benefits associated with reduced risky alcohol consumption and the cost of the intervention over the lifespan of a typical club. It was estimated that the intervention resulted in a cost reduction associated with short-term risky alcohol behaviour valued at approximately $\$ \mathrm{~A} 13.8$ million, or about $\$ \mathrm{~A} 3823$ for a typical club. Between 2011 and 2012, the return on investment for a typical club implementing the intervention was approximately \$A45600 in net present value terms, which equated to a benefit cost ratio of 4.2. Meaning, for every $\$ 1$ spent on implementing the programme, $\$ 4.20$ would be expected to be returned to the Australian economy.

Effects on poor diet, physical inactivity, obesity, risky alcohol use or tobacco use

Two trials reported on the effectiveness of implementation interventions on individual behaviours. One study found positive impacts on sports club members' diet choices $^{37}$ and the other study found a reduction in risky alcohol consumption. ${ }^{15}$

The proportion of club members who reported purchasing fruit and vegetable products from sports club canteens was presented by Wolfenden et al. ${ }^{37}$ This study found a significant increase in fruit and vegetable products being purchased among members from intervention clubs compared with members from control clubs (OR: $2.58 ; 95 \%$ CI 1.08 to $6.18 ; \mathrm{p}=0.033$ ). Additionally, there was a significant increase in the proportion of intervention club members who reported purchasing non-sugar-sweetened drinks compared with control club members (OR: $1.56 ; 95 \%$ CI 1.09 to $2.25 ; \mathrm{p}=0.015$ ).

A companion paper for the Kingsland et al's study ${ }^{15}$ reported on the proportion of club members who consumed alcohol at risky levels (defined as consuming five or more drinks on one occasion) at the club ground.
There was a significant between-group difference at follow-up $(\mathrm{p}=0.05)$ with risky alcohol consumption reduced from $27 \%$ to $19 \%$ among intervention club members between baseline and follow-up, compared with almost little change (25\% to $24 \%$ ), among control club members over this period (OR: $0.63 ; 95 \%$ CI 0.40 to 1.00 ).

\section{Reported adverse consequences}

To assess the potential adverse intervention impact on the sale of healthy foods and non-alcoholic beverages from the sports club canteen Wolfenden et al conducted Computer Assisted Telephone Interview (CATI) surveys with club representatives. ${ }^{37}$ Club representatives were asked to report the approximate total income from foods and non-alcoholic beverages over a 1-year period. After intervention there was no significant difference of mean annual revenue between intervention (\$A29 669, SD: \$A31 205) and control (\$A26 529, SD: \$A33 465) clubs $(\mathrm{p}=0.910)$.

A companion paper for the Kingsland $e t$ al's study, Wolfenden $e t a l^{44}$ also conducted CATI surveys to measure the impact the alcohol management intervention had on sports club revenue and membership. Club representatives were asked to report their clubs' approximate total income over the past year and the number of current club players, number of current senior teams and usual number of spectators attending senior home games (table 1). At follow-up, the number of players or senior teams was not significantly different between groups $\mathrm{p}=0.331$ and $\mathrm{p}=0.733$, respectively. There was however a significantly higher mean number of spectators at follow-up for intervention clubs than the control clubs $(\mathrm{p}=0.020)$. Finally, there was no significant difference in revenue between groups at follow-up $(\mathrm{p}=0.378)$, with a mean increase of \$A19356 for intervention clubs and a mean increase of \$A42617 for control clubs.

\section{DISCUSSION}

This review sought to assess the impact of strategies to improve the implementation of policies, practices or programmes in sporting organisations targeting poor diet, physical inactivity, obesity, risky alcohol use or tobacco use. The review identified just three trials meeting the inclusion criteria. Each trial reported improvement in at least one measure of policy or practice implementation. The findings of this review suggest that improvements in the implementation of health promotion policies and practice in the sporting clubs setting are possible. However, the presented research base is varied, and due to the limited number of studies and health risk behaviours covered, provides limited evidence to guide policy or practice. The overall quality of the body of evidence in the review was rated as very low across all GRADE domains. ${ }^{35}$

With few reviews examining the effectiveness of implementation strategies in community settings broadly, and no such trials identified within a review of the sports setting previously, ${ }^{26}$ contextualising the findings of this 
review is challenging. One similar review conducted in the child care setting ${ }^{21}$ synthesised 10 trials testing multicomponent implementation support strategies. This review found overall low-quality evidence to support the included trial effectiveness. ${ }^{19}$ Additionally, an effect size range of $0 \%-9.5 \%$ was found for the four trials measuring the proportion of child care services implementing a policy or practice. ${ }^{21}$ Furthermore, an additional review on the effectiveness of implementation strategies to support interventions in clinical settings reported the use of educational outreach visits resulted in a median effect size of 23\% in improving professional practice compared with controls. ${ }^{45}$ Additionally, the review found improvements to intervention practice compliance through educational meetings and workshops with a median effect size of $10 \%$, and the use of audit and feedback with a median effect size of just $1.3 \%$ relative to controls. ${ }^{45}$ The findings of this review suggest that multistrategic interventions may have the potential to improve the implementation of policies and practices targeting health behaviours. The common implementation strategies across the three trials were: educational materials, educational outreach visits and monitoring performance. Two of the included studies adopted a hybrid design, meaning that both implementation strategy outcomes and individual behavioural change outcomes were collected. ${ }^{46}$ Similar to other implementation reviews, ${ }^{21} 45$ this review found little evidence of the assessment or reporting of cost or cost-effectiveness of health-promoting policies or practices, with only one study including this as an outcome. Further, while club revenue was assessed as a potential adverse event in two trials, little consideration was given to a range of potential unintended adverse consequences to sporting organisations, their staff or players among included trials. As policymakers and practitioners weigh the beneficial effects of interventions (or implementation strategies) with their costs and risk of adverse effects, research to address this evidence gap is warranted.

The included studies should be considered with regard to the limitations. First, selection bias, performance bias and detection bias were considered to be high for one or more of the included studies. Furthermore, all trials included the use of self-report assessment of outcomes. Although high levels of corroboration between self-report and visual observations have been found in previous studies in licensed venues, the direct observation of practices, policies and the collection of food sales data would have provided more valid estimate of outcomes. Furthermore, the short intervention period in Naylor et al's study may have impacted sporting facilities degree of change. Both Kingsland et al and Wolfenden et al recruited only clubs from the four primary football codes in Australia. Finally, all included studies were conducted in Australia and Canada, both high-income countries, and no studies targeted tobacco use, obesity and physical inactivity. Therefore, the ability to generalise study findings to other sports codes, lower and middle-income countries and other health risk behaviours, where the operational and cultural contexts may differ, is unknown.

The potential methodological limitations of this review should be considered. As suggested for complex reviews of public health and health promotion interventions, ${ }^{23} \mathrm{a}$ search filter was employed when undertaking the initial search for this review. While rigorous searches were undertaken, terminology within the field of implementation is evolving. However unlikely, the potential of eligible studies being missed through the use of this search filter needs to be considered. Additionally, through the non-publication of studies with negative results or due to a lack of academic involvement in the evaluation of such strategy implementation, and limitation of other searching methods, potentially some studies may have been missed.

This review found only three studies examining the implementation of policy, practice or programme targeting alcohol use and healthy eating/obesity. Although the included studies reported increases in practice and policy implementation, it is difficult to determine the extent to which such effects are generalisable. Additionally, none of the included studies measured the long-term implementation of the practice or policy and therefore any long-term improvements to those health risk behaviours are unable to be determined. Thus, our ability to identify effective strategies or provide clear directions for future sports-based interventions is limited. Further controlled trials that employ high standards of methodology and are implemented in varying sports settings, with measures for long-term implementation, are required to strengthen the applicability of the evidence base. It would be potentially beneficial for a review to be conducted with an inclusion criterion aimed at capturing those studies (observational studies) which do not sit within the requirements of this review. Such a review may further inform the current body of evidence and strengthen the identification of research gaps in this area.

\section{Author affiliations}

${ }^{1}$ School of Medicine and Public Health, The University of Newcastle, Callaghan, New South Wales, Australia

${ }^{2}$ Population Health, Hunter New England, Wallsend, New South Wales, Australia ${ }^{3}$ School of Health Sciences, The University of Newcastle, Callaghan, New South Wales, Australia

${ }^{4}$ School of Public Health, The University of Sydney, Sydney, New South Wales, Australia

Acknowledgements The authors acknowledge the contribution of Debbie Booth from The University of Newcastle who provided guidance regarding the search strategy and conducted the search of electronic databases.

Collaborators Debbie Booth.

Contributors TM led the review, contributed to search design, conducted study search, and undertook additional screening, risk of bias assessment, GRADE assessment and study synthesis. LKC contributed to search design, conducted study screening, risk of bias assessment, and contributed to manuscript preparation. RW conducted study screening and contributed to manuscript preparation. MK, AB, JW, CR and CMW contributed to manuscript preparation. TCM contributed to risk of bias assessment and to manuscript preparation. SLY conducted EPOC classification and contributed to manuscript preparation. LW 
designed the study search, conducted GRADE assessment and contributed to study synthesis and manuscript preparation.

Funding The Australian Prevention Partnership Centre provided funding support for this review.

Competing interests MK, LW and JW authored two of the studies included in this review. For these studies, data were extracted and risk of bias was conducted by review authors who were not directly involved in these studies. The authors have not received any benefit, in cash or in kind, any hospitality or any subsidy or any other source perceived to have an interest in the outcome of this review.

Patient consent Not required.

Provenance and peer review Not commissioned; externally peer reviewed.

Data sharing statement As this is a systematic review the authors do not have access to all original data sets of the included studies. However, all data available to the authors can be obtained by contacting the corresponding author.

Open access This is an open access article distributed in accordance with the Creative Commons Attribution Non Commercial (CC BY-NC 4.0) license, which permits others to distribute, remix, adapt, build upon this work non-commercially, and license their derivative works on different terms, provided the original work is properly cited, appropriate credit is given, any changes made indicated, and the use is non-commercial. See: http://creativecommons.org/licenses/by-nc/4.0/.

\section{REFERENCES}

1. Lim SS, Vos T, Flaxman AD, et al. A comparative risk assessment of burden of disease and injury attributable to 67 risk factors and risk factor clusters in 21 regions, 1990-2010: a systematic analysis for the Global Burden of Disease Study 2010. Lancet 2012;380:2224-60.

2. Institute for Health Metrics and Evaluation. Global burden of disease study 2013. 2013 www.healthdata.org/gbd

3. World Health Organization. Global status report on noncommunicable diseases 2014. 2014 http://www.who.int/nmh/ publications/ncd-status-report-2014/en/

4. Corti B, Brimage G, Bull F, et al. Health-promoting sport, arts, and race settings: new challenges for the health sector: National Health and Medical Research Council, Australian Government Publishing Service, 1996.

5. Crisp BR, Swerissen H. Critical processes for creating healthpromoting sporting environments in Australia. Health Promot Int 2003;18:145-52.

6. Kokko S, Kannas L, Villberg J. The health promoting sports club in Finland-a challenge for the settings-based approach. Health Promot Int 2006;21:219-29.

7. Kokko S. Sports clubs as settings for health promotion: fundamentals and an overview to research. Scand J Public Health 2014;42(15 Suppl):60-5.

8. Sports England. Active People Survey 10Q2 April 2015 to March 2016. 2016 https://www.sportengland.org/media/10745/1x30_ overall_factsheet_aps10q2.pdf

9. Australian Sports Commission. AusPlay participation data for the sport sector: summary of key national findings October 2015 to September 2016 data. 2016 https://www.clearinghouseforsport.gov. au/_data/assets/pdf_file/0011/735239/34648_AusPlay_summary_ report_accessible2.pdf

10. Hunt $\bar{K}$, Wyke S, Gray CM, et al. A gender-sensitised weight loss and healthy living programme for overweight and obese men delivered by Scottish Premier League football clubs (FFIT): a pragmatic randomised controlled trial. Lancet 2014;383:1211-21.

11. Gray CM, Hunt K, Mutrie N, et al. Weight management for overweight and obese men delivered through professional football clubs: a pilot randomized trial. Int J Behav Nutr Phys Act 2013;10:121.

12. Wyke S, Hunt K, Gray CM, et al. Football Fans in Training (FFIT): a randomised controlled trial of a gender-sensitised weight loss and healthy living programme for men - end of study report. Public Health Res 2015;3:1-130.

13. Dubuy V, De Cocker K, De Bourdeaudhuij I, et al. Evaluation of a real world intervention using professional football players to promote a healthy diet and physical activity in children and adolescents from a lower socio-economic background: a controlled pretest-posttest design. BMC Public Health 2014;14:457.

14. Wagner P, Brehm W, Sygusch R. The seven-sequence intervention: sedentary adults on their way to fitness and health. Res Sports Med 2004;12:265-82.

15. Kingsland M, Wolfenden L, Tindall J, et al. Tackling risky alcohol consumption in sport: a cluster randomised controlled trial of an alcohol management intervention with community football clubs. J Epidemiol Community Health 2015;69:993-9.

16. Marcus $\mathrm{BH}$, Albrecht AE, King TK, et al. The efficacy of exercise as an aid for smoking cessation in women: a randomized controlled trial. Arch Intern Med 1999;159:1229-34.

17. Victoria State Government - Health and Human Services. Healthy choices: policy guidelines for sport and recreation centres, 2012.

18. Government of Alerta. Alberta nutrition guidelines for children and youth, 2012.

19. Drygas W, Ruszkowska J, Philpott M, et al. Good practices and health policy analysis in European sports stadia: results from the 'Healthy Stadia' project. Health Promot Int 2013;28:157-65.

20. Lenk KM, Toomey TL, Erickson DJ, et al. Alcohol control policies and practices at professional sports stadiums. Public Health Rep 2010;125:665-73.

21. Wolfenden L, Jones J, Williams CM, et al. Strategies to improve the implementation of healthy eating, physical activity and obesity prevention policies, practices or programmes within childcare services. Cochrane Database Syst Rev 2016;10:CD011779.

22. Rabin BA, Glasgow RE, Kerner JF, et al. Dissemination and implementation research on community-based cancer prevention: a systematic review. Am J Prev Med 2010;38:443-56.

23. Higgins JP, Green S. Cochrane handbook for systematic reviews of interventions: John Wiley \& Sons; The Cochrane Collaboration, 2011.

24. Moher D, Shamseer L, Clarke M, et al. Preferred reporting items for systematic review and meta-analysis protocols (PRISMA-P) 2015 statement. Syst Rev 2015;4:1.

25. Effective Practice and Organisation of Care. EPOC taxonomy. 2015 https://epoc.cochrane.org/epoc-taxonomy

26. Priest N, Armstrong R, Doyle J, et al. Policy interventions implemented through sporting organisations for promoting healthy behaviour change. Cochrane Database Syst Rev 2008:CD004809.

27. Dobbins M, Husson H, DeCorby K, et al. School-based physical activity programs for promoting physical activity and fitness in children and adolescents aged 6 to 18. Cochrane Database Syst Rev 2013;2:CD007651.

28. Guerra PH, Nobre MR, da Silveira JA, et al. School-based physical activity and nutritional education interventions on body mass index: a meta-analysis of randomised community trials - project PANE. Prev Med 2014;61:81-9.

29. Jaime PC, Lock K. Do school based food and nutrition policies improve diet and reduce obesity? Prev Med 2009;48:45-53.

30. Waters E, de Silva-Sanigorski A, Burford BJ, et al. Interventions for preventing obesity in children. Cochrane Database Syst Rev $2011 ; 165$

31. Thomas RE, McLellan J, Perera R. School-based programmes for preventing smoking. Cochrane Database Systematic Review 2013;4:CD001293.

32. Foxcroft DR, Ireland D, Lister-Sharp DJ, et al. Primary prevention for alcohol misuse in young people. Cochrane Database Systematic Review 2002;3.

33. Rabin BA, Brownson RC, Haire-Joshu D, et al. A glossary for dissemination and implementation research in health. $J$ Public Health Manag Pract 2008;14:117-23.

34. Wolfenden L, Nathan NK, Sutherland R, et al. Strategies for enhancing the implementation of school-based policies or practices targeting risk factors for chronic disease: The Cochrane Library, 2017

35. Guyatt GH, Oxman AD, Schünemann HJ, et al. GRADE guidelines: a new series of articles in the Journal of Clinical Epidemiology. J Clin Epidemiol 2011;64:380-2.

36. Kingsland M, Wolfenden L, Tindall J, et al. Improving the implementation of responsible alcohol management practices by community sporting clubs: A randomised controlled trial. Drug Alcohol Rev 2015;34:447-57.

37. Wolfenden L, Kingsland M, Rowland BC, et al. Improving availability, promotion and purchase of fruit and vegetable and non sugar-sweetened drink products at community sporting clubs: a randomised trial. Int J Behav Nutr Phys Act 2015;12:35.

38. Naylor P-J, Olstad DL, Therrien S. An intervention to enhance the food environment in public recreation and sport settings: a natural experiment in British Columbia, Canada. Child Obes 2015;11:364-74.

39. Damschroder LJ, Aron DC, Keith RE, et al. Fostering implementation of health services research findings into practice: a consolidated framework for advancing implementation science. Implement Sci 2009;4:50.

40. New South Wales Health. A framework for building capacity to improve health. Gladsville: Better Health Centre, 2001.

41. Kingsland $M$, Wolfenden $\mathrm{L}$, Rowland $\mathrm{BC}$, et al. A cluster randomised controlled trial of a comprehensive accreditation intervention to 
reduce alcohol consumption at community sports clubs: study protocol. BMJ Open 2011;1:bmjopen2011000328.

42. Stokols D. Translating social ecological theory into guidelines for community health promotion. Am J Health Promot 1996;10:282-98.

43. KPMG. Economic evaluation of the Good Sports program: Australia Drug Foundation, 2013.

44. Wolfenden L, Kingsland M, Rowland B, et al. The impact of alcoho management practices on sports club membership and revenue. Health Promot J Austr 2016;27:159-61.

45. Lau R, Stevenson F, Ong BN, et al. Achieving change in primary care-effectiveness of strategies for improving implementation of complex interventions: systematic review of reviews. BMJ Open 2015;5:e009993.

46. Wolfenden L, Williams CM, Wiggers $\mathrm{J}$, et al. Improving the translation of health promotion interventions using effectiveness-implementation hybrid designs in program evaluations. Health Promot $J$ Austr 2016;27:204-7.

47. Naylor PJ, Vander Wekken S, Trill D, et al. Facilitating healthier food environments in public recreation facilities: Results of a pilot project in British Columbia, Canada. Journal of Park and Recreation Administration 2010;28. 